\title{
Bridging the Health Divide: Use of Mobile Phone Technology for Health Care Service Delivery in Odisha
}

\section{Sahoo $\mathbf{S}^{*}$}

Department of Journalism and Mass Communication, Berhampur University, Ganjam, Odisha, India

\begin{abstract}
Access to health care has always remained a major challenge across most of the developing countries. In India, where nearly 68.85 per cent of the population reside in rural areas, public health service faces many technological and operational challenges. Similarly, Odisha, being considered a poor state and having a rural population of 83.31 per cent also bears the brunt of health divide to a great extent. No doubt, the government has initiated several schemes to address public health issues, but lack of awareness among the stakeholders, inadequate physical infrastructure and geographical barriers have affected a lot in health care delivery. However, rapid adoption of mobile telephone technology in rural areas has brought forth newer possibilities in health communication to bridge the health divide. According to the data given by TRAI, Odisha has a tele-density of 81.08 per cent as on July 31,2017 . Hence, mobile communication has a greater potentiality to be exploited as dependable and effective ICT media for public health. At the same time, the growing sophistication of the mobile networks - offering high speed data and cheaper but powerful handsets - has come with the possibility of greater personalisation and citizen-focused public health and medical care. This paper aimed to explore the multiple roles and effectiveness of mobile communication for the delivery function of public health programmes related to child and maternal health care. Apart from a discussion on audio-visual contents and mobile applications, the study, based on literature and interviews, has also analysed how the health services is improved by using prompts and reminders through voice calls and text messages for the health workers.
\end{abstract}

Keywords: Public Health; mHealth; Mobile phone technology; ICT; Health Communication

\section{Introduction}

When we walk around any city or village, we can see different sized houses, different types of vehicles, and different types of activities going on. These differences are the indicators of disparity among individuals in terms of wealth, assets, income or some other criteria. What the differences we see around us in economic levels, is also applicable on a larger scale to the nations of the world. But development is not always calculated only on the basis of economic development or in terms of per capita income output, rather it is based on the complex cultural and environmental factors and their interactions. Hence, it is not at all surprising that countries with same average incomes may differ substantially when it comes to the standard of living of the citizens - employment opportunities, education, health care, availability of clean air and drinking water, threat of crime etc. And in modern day society, the importance of health care can be gauged as good health is considered one of the fundamental rights of an individual [1].

World Health Organisation (WHO) in its fact sheet reiterates that the highest attainable standard of health is a fundamental right of every human being. "The right to health includes access to timely, acceptable and affordable health care of appropriate quality." No doubt, the overall population health indicator has improved a lot, but the experience is not very equal everywhere, or for all. Particularly, in the developing countries the equal access to health care has still remained a major challenge. Out of many factors, rural-urban disparities have always been a cause of concern across these third world countries creating health divide. When preventive or primary care is missing, a mild sickness may turn into a complicated condition which might require emergency attention, attracting much higher expenses [2-4].

India, still predominantly rural with nearly 68.85 per cent of the population residing in rural areas, faces many technological and operational challenges in delivering proper health care service. About
80 per cent of health infrastructure, medical manpower and other health resources are urban centric. Hence, the rural health care is dependent on limited government facilities and private initiatives. The quality of infrastructure is poor; thereby people are dependent on cities for quality health care access. It is obvious that the maternal and infant mortality number is high in rural areas due to lack of emergency and timely health care at the local health care centres.

Like the popular saying - India lives in villages, Odisha also lives in villages. As per 2011 census, 83.31 per cent of the total population of the state lives in rural areas. It is ranked third in terms of Scheduled Tribe (ST) population in the country. No doubt, the government has initiated several schemes to address public health issues, but lack of awareness among the stakeholders, inadequate physical infrastructure and geographical barriers have affected a lot in health care delivery [5].

In the absence of the complex functioning of three-tier referral systems and accessibility to secondary or tertiary health services, Government has envisaged using the telemedicine systems to improve health care at primary level. But it is limited to a few tertiary care public and private hospitals. In the meantime, tele-consultation under telemedicine system is also very cost effective [6,7].

In this context, a low cost ICT alternative is the need of the

*Corresponding author: Sahoo S, Department of Journalism and Mass Communication, Berhampur University, Ganjam, Odisha, India, Tel: +919692434667; E-mail: s.sudarsansahoo@gmail.com

Received May 22, 2018; Accepted September 17, 2018; Published September 24 2018

Citation: Sahoo S (2018) Bridging the Health Divide: Use of Mobile Phone Technology for Health Care Service Delivery in Odisha. J Mass Communicat Journalism 8: 391. doi: 10.4172/2165-7912.1000391

Copyright: (c) 2018 Sahoo S. This is an open-access article distributed under the terms of the Creative Commons Attribution License, which permits unrestricted use, distribution, and reproduction in any medium, provided the original author and source are credited. 
hour. Application of mobile phone and wireless technology has the potential to meet the challenge. In India, people have more access to mobile devices than to electricity, clean piped water and road systems. According to Telecom Regulatory Authority of India (TRAI), India has some 1183.04 million mobile phone subscribers by the end of September 2017 with a rural subscription of 498.28 million (nearly 42 per cent). The overall tele-density is $91.56 \%$, while the urban and rural shares are $57.88 \%$ and $42.12 \%$ respectively. In Odisha, the overall tele-density is $80.79 \%$. Therefore, mobile communication has a greater potentiality to be exploited as dependable and effective ICT media for public health. At the same time, the growing sophistication of the mobile networks - offering high speed data and cheaper but powerful handsets - has come with the possibility of greater personalisation and citizen-focused public health and medical care [8]. Moreover, mobile phone as a media is now comparable to the popular media like radio and television which were traditionally used for spreading health care awareness for decades. Perhaps it will enjoy the status of the most accessible ICT tool in rural areas for many years to come.

The spread of mobile technology and its priority in health care has created a new field of eHealth i.e., mHealth (mobile health). mHealth has the capability to provide individually tailored and customised services to underserved people comparatively at a low cost. This paper aims to discuss the key application areas where mobile phone technology can bridge the health divide. It also gives a comprehensive picture of the Government initiatives and implementations for the mHealth in the context of child and maternal health care [9].

\section{What is mhealth?}

mHealth is the use of telecommunication and multimedia technologies with integration of mobile phones and wireless health care delivery system. According to WHO's Global Observatory for eHealth, "mHealth is medical and public health practice supported by mobile devices, such as mobile phones, patient monitoring devices, personal digital assistants and wireless devices." mHealth capitalizes on mobile phone's basic functionalities i.e., voice and short messaging service (SMS) as well as the complex utilities and applications like general packet radio service (GPRS), global positioning system (GPS), Bluetooth technology and $3 \mathrm{G}$ or $4 \mathrm{G}$ mobile telecommunication systems. Although development of mHealth is in its early stage, the success of the mHealth applications and programs in the developing world in improving health care delivery is well documented [10].

\section{mHealth Worldwide}

A survey by WHO in 2011 indicates that mHealth initiatives have been taken in many countries. The mHealth adoption rate in North America, South America and Southeast Asia is highest, while in Africa it is lowest. At least $51 \mathrm{mHealth}$ programs that are operational in 26 developing countries have been listed in a study by United Nations and Vodafone Foundation. These programs basically focus on emergency medical services, point of care services and health information systems, drug adherence and disease surveillance, data collection and remote monitoring etc. Some popular mHealth initiatives worldwide are discussed below.

In United States, mHealth applications like Gluco Phones (monitor blood glucose levels), Text4Baby (handling issues during pregnancy) and Heal the Trax (virtual health assistant) are very popular. In Indonesia, people chat and consult with physicians through interfacing applications like Dokita (our doctor) and DokterGratis (free doctor). Vaccine workers in Bangladesh use mTikka application for infant registration that automatically generate vaccination schedules and send reminders to the parents [11].

\section{mHealth Initiatives in India}

In India, mHealth is initially used for maternal and child tracking system where messages were sent to Janani Suraksha Yojana beneficiaries and the Accredited Social Health Activist (ASHA) workers. System operated automatic messages helped in improvement of service delivery. The government of India, in 2012, introduced NIKSHAY, a program to monitor TB patients through SMS services.

In 2016, Indian government has launched four mHealth initiatives - Kilkari, Mobile Academy, M-Cessation and TB Missed Call - as a part of its Digital India programme. Kilkari means a baby's gurgle. Under Kilkari programme, a woman receives free, weekly and timeappropriate 72 audio messages about pregnancy, child birth and child care from second trimester of pregnancy till the child becomes one year old. It is launched in Odisha, Jharkhand, Uttar Pradesh and some parts of Madhya Pradesh and Rajasthan.

Mobile Academy is a training course developed by BBC Media Action to refresh and expand the knowledge and communication skills of the Accredited Social Health Activists (ASHAs). The audio course is delivered via Interactive Voice Response Service (IVRS) - a technology which can be accessed from any type of mobile handsets. It enables the community health workers to complete the course anywhere at any pace and at times they find convenient.

M-Cessation targets to reach out to those who want to quit tobacco use and sends text messages via mobile phones to support them towards successful quitting. Against traditional methods, M-Cessation stands superior and cost-effective. For the first time in the world, such a two way service is being initiated as part of any mHealth initiative. TB Missed Call initiative enables a beneficiary to get information; counselling and treatment support services for TB by giving a missed calls or call to a toll free number [12].

Besides, the National Health Portal of India in its website provides a list of 50 mobile applications and 24 websites under mHealth category which can be used by the citizens for health care.

\section{Opportunities of mhealth in Odisha}

\section{Odisha: A fact file}

According to the Census 2011, the state of Odisha, with an area of 155,707 sq. $\mathrm{km}$. has a population of 41 million. It has 30 districts, 314 blocks and 51349 villages. The population of the state forms 3.47 percent of the total population of India. At least 83.31 per cent of the total population of the state lives in rural areas. It is ranked third in terms of Scheduled Tribe (ST) population in the country. The state stands at $11^{\text {th }}$ rank as per population and $9^{\text {th }}$ in terms of geographical area in the country. The average population density is about 260 per sq. $\mathrm{km}$. The overall sex ratio of the state is 978 females per 1000 males. It is lowest (916) in Nayagarh district while the highest (1048) is in Rayagada district. The literacy rate is 73.45 (64.36 per cent for females and 82.40 per cent for males).

In Odisha, except the District Headquarters Hospitals, there are 377 community health centres (CHCs), 1305 primary health centres (PHCs) and 6688 subcentres. As many as 8245 female health workers/ ANMs are in position to deliver health services at subcentres and PHCs. In 2013 , the infant mortality rate was 51 and the maternal mortality rate is 222 . 


\section{Exploring the opportunity of mobile phone in health care}

In Odisha, the frontline health workers always face several challenges in delivering quality health care on time. However, using a variety of technologies like IVRS, text SMS, MMS, and multimediaenabled applications on smart phones, the potentials of mHealth solutions can be explored to support the front line health workers in five verticals. These verticals are:

1. Self-learning/Refresher Training

2. Patient Registration and Monitoring

3. Scheduling and Task Management

4. Awareness and Counselling

5. Clinical Decision and Assessment Support.

We will discuss how far the potentiality of mobile phones is being exploited in delivering child and maternal health care in Odisha in the context of these five verticals.

Self-learning/refresher training: mHealth solutions help the frontline health workers learn by going through mobile-friendly training modules with digital or audio contents. These programmes usually assess the trainees using tools such as audio or digital quizzes and tests. Odisha Chief Minister Naveen Patnaik launched mobile health services - Mobile Kunji and Mobile Academy - in Rourkela on February 23, 2014 in an effort to reduce Mother Mortality Rate (MMR) and Infant Mortality Rate (IMR). Mobile Academy, which is developed by BBC Media Action, is a self-learning audio programme for ASHAs as they can refresh their knowledge and develop their communication skills through IVRS. This course can be accessed from any mobile handset. In this context, the comment of head of BBC Media Action India, Sara Chamberlain's comment is worth mentioned. She said, "We learned that ASHAs tend to listen to the modules after lunch or after dinner and felt that self-learning fit better into their schedule versus interrupting their schedule for offsite government training." However, during an interaction with some of the frontline workers in Nayagarh district of Odisha, it was found that they are yet to get any orientation about Mobile Academy by the concerned department.

Patient registration and monitoring: $m$ Health gives a scope to the health workers to complete the registration process on the field itself instantly through specified mobile applications. It creates real-time data and reduces the chance of delay in solving gaps in delivering essential medical help due to paper-based reporting backlog of information. The efficacy of cell phone-based registration was successfully demonstrated during the implementation of mSakhi project in Maharashtra which aims to collect data, monitor and evaluate the antenatal and postnatal health care and nutrition of infants up to six months. However, such types of mHealth initiatives are yet to be taken in Odisha. But community health workers collect mobile phone numbers mandatorily during the registration of the pregnant women, new-borns and other beneficiaries along with other basic information like Aadhaar or National Population Register number. As a result, the beneficiaries receive SMS and IVR calls to get updates regarding health care.

Scheduling and task management: The scheduling and task management solutions of mHealth creates daily tasks for the community health workers based on the health requirements derived from the patient data base. These mobile solutions feed the central database and also allow the supervisors to stay informed about the completed tasks. For example, CARE India, an NGO, has launched an Integrated Family
Health Initiative application (CCS: Continuum of Care Services) in Bihar integrating with the counselling and awareness tool of $\mathrm{BBC}$ Media Action. The CCS application informs the Anganwadi workers and the ASHAs about to which women they should visit and what the topics they should cover are. The application tracks the health workers' activity and sends data to their supervisor as well as the central server. However, such type of initiatives is yet to be implemented in Odisha.

Awareness and counselling: Traditionally, the community health workers use paper-based job aids like text-heavy reference materials, bulky flipbooks and complex newborn care checklists. These materials are difficult to use and adopt. The beneficiaries also find the bulky display materials less interesting. But BBC Media Action's Mobile Kunji (in Hindi it means 'guide' or 'key'), is portable, multimedia enabled and interactive easing the difficulties of the health workers in counselling the families. The Mobile Kunji is a combination of laminated cards on a steel ring. The cards, which are in shape of mobile phones, carry illustrations, supporting arguments and key messages about maternal and child health and a unique number code on each page. After reading out the messages to the beneficiary, the health worker dials the code which corresponds to a specific audio message through IVRS. The audio messages are the recorded voice of Dr Anita. In Odisha, Mobile Kunji service has been launched since 2014, but it has not been widely incorporated so far.

Meanwhile, the Central Government has also initiated Kilkari project under which Odisha is a beneficiary. It delivers free, weekly and timely audio messages directly to the registered mobile number about pregnancy, child birth and child development. At least 72 messages have been designed under the project which reaches the beneficiaries periodically. Besides, the pregnant women and mothers having infants also receive SMS from the automated systems after the registration of their mobile numbers under different schemes like Janani Suraksha Yojana and Mamata.

Clinical decision and assessment support: By going through the suggestions and the step-by-step guide of mHealth applications, the frontline health workers gradually develop their skills in identification, management and referral of the sick mothers and newborns. The IntraHealth study on mShakti application affirms that ASHAs who used the support of the application were better on patient diagnosis, treatment and referrals. Hence, in Odisha, where a large number of people live off the city areas, taking clinical decisions for the frontline health workers can be easier throug $\mathrm{h}$ mHealth applications.

The National Health Mission, Odisha, under its Mission Connect scheme, has created a Closed User Group (CUG) of designated health service providers of Department of Health and Family Welfare. At least 55,605 CUG members are provided free BSNL SIM cards. They can recharge with any special amount starting from Rs. 99, and thereby they can enjoy unlimited voice calls within the CUG. It helps them to take clinical decisions and share their views to assist each other virtually round the clock. The ASHAs also call 108 Ambulance service on behalf of the anticipating mothers to go for institutional delivery.

\section{Challenges in Incorporating mhealth}

There are a lot of challenges to incorporate mHealth in Odisha. Usually mobile applications are built in English for display, transfer, input and data processing. But using local languages or dialects in the mobile application invites tough challenges. Similarly, integrating with medical devices to mobile application requires sophisticated technology. It also needs coordination of multidisciplinary fields like 
psychology, medical, business and computer science. On the other hand, the public health divisions of the central and state governments are also lacking adequate capacity to design and implement mobile health in the field.

\section{Conclusion}

Different studies and projects incorporated in third world countries have proved the effectiveness of mHealth initiatives in different problem areas in resource-poor setting. In Indian setting also, a number of mHealth initiatives like mDristhi and mShakti have been applied impressively. In Odisha, though the implementation of mHealth is not very impressive, but the response is good. Proper implementation of mHealth can provide accurate and real-time data which will help monitor and track the health and development of women and children. Hence, it can be said that mHealth has the real potency to bridge the health divide of Odisha which is facing a lot of difficulties in health service delivery.

\section{References}

1. Chakrabarty S (2014) Household expenditure on health care in urban Odisha: An empirical study of Cuttack city. PhD Thesis. KIIT University. Bhubaneswar.

2. Goli S (2012) Rural-Urban Divide in Health Status. Geography and You, pp: 54-57.
3. World Health Organisation. (2015). Health and Human Rights. Fact Sheet No 323.

4. National Commission on Macroeconomics and Health MHFW, Gol. (2005) Report of the National Commission on Macroeconomics and Health. New Delhi.

5. Nighat A (2013) Regional disparities in economic development in post-reform period. PhD thesis. Aligarh Muslim University.

6. Garai A (2011) Role of mHealth in rural health in India and opportunities for collaboration. In, ICCP Technology Foresight Forum: Developments in Mobile Communications, Paris.

7. TRAI (2017) High Lights of Telecome Subscription Data. [Press Release].

8. Ahamed F ,et al. (2017) Scope of mobile health in Indian health care systemthe way forward. International Journal of Community Medicine and Public Health 4: 875-881.

9. World Health Organisation (2011) mHealth: New horizons for health through mobile technologies: second global survey on eHealth. Switzerland.

10. United Nations Foundation. (2009). mHealth for Development: The Opportunity of Mobile Technology for Healthcare in the Developing World. Washington, D.C. and Berkshire, UK: Vital Wave Consulting.

11. mPowering Frontline Health Workers, United Nations Foundation \& Qualcomm Wireless Reach. Opportunities to Improve Maternal, Neonatal and Child Health in India through Smart Phones and 3G Connectivity Solutions. [A report].

12. Mobile health services launched in Odisha. (2014, February 27). 